\title{
Quantification de l'analyse en profondeur par spectrométrie d'électrons Auger et pulvérisation ionique : caractérisation des interfaces des films multicouches $\mathrm{Cu}-\mathrm{Co}$
}

\author{
Françoise Basile, Jean Bergner, Claude Bombart, Pascale Nallet, \\ Elisabeth Chassaing et Gérard Lorang \\ Centre d'études de Chimie Métallurgique $\left(^{*}\right), 15$ rue G. Urbain, \\ 94407 Vitry/Seine Cedex, France
}

(Reçu le 3 juillet 1997, Accepté le 20 novembre 1997)

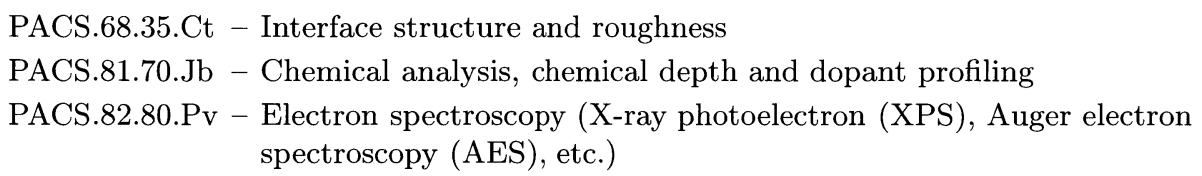

\begin{abstract}
Depth profiling analysis by AES combined with ion sputtering is employed for the characterization of $\mathrm{Co}-\mathrm{Cu}$ multilayer interfaces prepared either by $\mathrm{RF}$ sputter deposition or by electrodeposition. Depth concentration profiles are derived from Auger profiles with appropriate corrections of elemental sensitivities, escape lengths of Auger electrons and from changes of the sputter rate occuring at interfaces with composition; they are expressed as atomic densities which can provide significant atomic balances, film thicknesses and depth resolution measurements. Microroughness induced by sputtering is widely improved by the Zalar rotation method during etching, so the remaining contribution $(7-10 \mathrm{~nm})$ to the interface broadening mainly consists with atomic mixing whereas important resolution losses $(\Delta Z>20 \mathrm{~nm})$ observed in electrodeposited layers as well as in sputtered materials at $\mathrm{Co} \rightarrow \mathrm{Cu}$ interfaces can be attributed to the sample preparation rather to the sputtering process itself.
\end{abstract}

Résumé. - Les interfaces de matériaux multicouches $\mathrm{Cu}-\mathrm{Co}$ préparés par pulvérisation cathodique ou par électrodéposition sont caractérisées analytiquement par spectrométrie d'électrons Auger (AES) en combinaison avec l'abrasion ionique. La microrugosité induite par le bombardement d'ions est minimisée par la méthode de rotation "Zalar" des échantillons. L'approche quantitative utilisée prend en compte les sensibilités élémentaires, corrige les profils Auger de la profondeur d'échappement des électrons Auger et de l'évolution de la vitesse d'érosion avec la composition pour conduire aux profils de concentration sur lesquels est mesurée la résolution en profondeur aux interfaces $\Delta Z$. La largeur des interfaces $(\Delta Z=7-10 \mathrm{~nm})$ peut être alors globalement attribuée au mixage ionique d'atomes. Ainsi les pertes importantes de résolution $(\Delta Z>20 \mathrm{~nm})$ observées à certaines interfaces $(\mathrm{Co} \rightarrow \mathrm{Cu})$ paraissent imputables aux conditions d'élaboration de ces matériaux plutôt qu'aux effets induits par le bombardement d'ions.

$\left({ }^{*}\right)$ CNRS 15 


\section{Introduction}

La "résolution en profondeur" est le critère retenu pour caractériser la largeur des interfaces dans les profils d'analyse en profondeur obtenus par abrasion ionique. Selon la norme ASTM E1438-91 définie par le comité E-42 relatif aux analyses de surfaces [1], elle est mesurée par "la quantité de temps $\Delta t$ requise pour que le signal de l'un des éléments majeurs du film soit réduit de 84 à $16 \%$ de son intensité moyenne dans le film" sur les profils donnant l'évolution de l'intensité des signaux Auger en fonction du temps d'érosion. À des fins quantitatives et comparatives, la résolution en profondeur doit être exprimée en profondeur équivalente érodée $\Delta Z$, aussi convient-il de convertir $\Delta t$ en $\Delta Z$ grâce à la connaissance des vitesses d'érosion $Z^{*}$ dans les deux matériaux en vis-à-vis à l'interface. Celles-ci sont déterminées dans des films d'épaisseurs connues constitués par ces deux matériaux purs. Toujours selon cette norme ASTM, $Z^{*}$ est alors définie comme le rapport de l'épaisseur connue $Z$ du film par le temps d'érosion nécessaire pour atteindre à l'interface une intensité moitié de celle des plateaux $\left(t_{50 \%}\right)$. En règle générale, les vitesses d'érosion diffèrent d'un matériau à l'autre, aussi la conversion des temps d'érosion en profondeurs érodées n'est-elle plus linéaire au passage des interfaces où la vitesse d'érosion évolue en fonction de la composition. À ce stade de la quantification, cette évolution peut être prise en compte de manière approchée selon une procédure décrite par Hofmann [2,3]. Les performances du dispositif d'analyse et d'érosion sont imposées par la géométrie du système, par les caractéristiques du canon à ions et des films à analyser, etc. Elles doivent être établies à l'aide de couches et d'interfaces bien définies. Les films anodiques d'oxyde de tantale sur tantale aux interfaces abruptes constituent les matériaux de référence pour procéder à ce type de calibrage $[4,5]$.

Nombreux sont les facteurs liés au bombardement d'ions qui limitent la résolution en profondeur [2]. Parmi ceux-ci, la microrugosité induite par une pulvérisation unidirectionnelle constitue un élément dominant. Selon la méthode initiée par Zalar [6,7], elle peut être fort heureusement minimisée par la rotation des échantillons pendant l'abrasion qui, à l'état stationnaire de la pulvérisation $(Z>30 \mathrm{~nm})$, rend aussi la résolution en profondeur indépendante de la profondeur érodée $[8,9]$. Cette méthode sera mise à profit pour affiner l'analyse des interfaces des matériaux multicouches $\mathrm{Cu}-\mathrm{Co}$ dont l'intérêt majeur est de présenter des propriétés de magnétorésistance géante [10] qui paraissent particulièrement influencées par la structure des interfaces et par leur mode d'élaboration [11].

La profondeur d'échappement des électrons Auger contribue aussi à l'élargissement des interfaces observé sur les profils Auger. Il est intrinsèque à la technique analytique utilisée et au couple d'éléments étudiés. Sa contribution doit être prise en compte au terme de la quantification de l'analyse en profondeur avec la conversion des intensités Auger en densités atomiques [12,13]. La résolution en profondeur $\Delta Z$ mesurée sur ces profils de concentration peut désormais être considérée comme représentative de la contribution du mixage ionique à l'élargissement des interfaces [14]. Ce travail avait pour objectif d'estimer cette contribution dans nos conditions expérimentales pour le système $\mathrm{Cu}-\mathrm{Co}$ afin de pouvoir la distinguer des autres causes de déformation des interfaces comme celles pouvant survenir pendant d'élaboration de ces matériaux.

\section{Dispositif expérimental et préparation des échantillons}

L'analyse des films multicouches est réalisée par spectrométrie d'électrons Auger à l'aide d'un analyseur à miroir cylindrique (CMA, Cameca OPC-105) et d'un canon à électrons coaxial fonctionnant sous $2,5 \mathrm{keV}$ d'énergie et $400 \mathrm{nA}$ d'intensité primaires. L'incidence du faisceau d'électrons est de $30^{\circ}$ par rapport à la normale au plan de l'échantillon. L'acquisition des spectres s'effectue dans le mode dérivé avec $4 \mathrm{~V}$ de tension de modulation conduisant à une 
résolution relative en énergie $\Delta E / E$ de $0,28 \%$. Les intensités normalisées des transitions Auger du cuivre $(\mathrm{Cu}-\mathrm{LVV}$ à $918 \mathrm{eV})$ et du cobalt $(\mathrm{Co}-\mathrm{LVV}$ à $775 \mathrm{eV})$ en fonction du temps d'érosion constituent les profils Auger utilisés pour accéder, après quantification de l'analyse en profondeur, aux profils de concentrations. Comme la transition LMM du cuivre à $774 \mathrm{eV}$ interfère avec la transition LVV du cobalt à $775 \mathrm{eV}$, la contribution du cuivre dans Co-LVV estimée à $22 \%$ de l'intensité de la transition $\mathrm{Cu}-\mathrm{LVV}$ sera soustraite pour la présentation des profils de cobalt.

L'abrasion ionique est effectuée à l'aide d'un canon à ions (Riber CI-50 RB) opérant sous pompage différentiel $\left(5 \times 10^{-8}\right.$ mbar de krypton dans la chambre d'analyse, $3 \times 10^{-6}$ mbar dans le canon). Le faisceau d'ions krypton utilisé présente les caractéristiques suivantes : énergie primaire de $3 \mathrm{keV}, 57^{\circ}$ d'incidence, intensité en courant transmis de $60 \mathrm{nA}$ sur du tantale pur, taille du cratère : $0,04 \mathrm{~cm}^{2}$. Il dispose d'un système de balayage permettant de visualiser l'échantillon de manière à faire coïncider le centre du cratère d'érosion $\left(0,04 \mathrm{~cm}^{-2}\right)$ avec la plage analysée par le faisceau d'électrons [18].

La rotation des échantillons autour de l'axe normal au plan d'analyse est assurée par un moteur pas-à-pas fonctionnant sous ultra-vide à 1,5 tours par minute. La plage d'analyse se situe obligatoirement à l'emplacement de l'axe de rotation. Celui-ci est repéré par le centre de la trace circulaire laissée sur la couche de pollution superficielle par le faisceau d'électrons focalisé sur l'échantillon en cours de rotation.

Les couches d'épaisseurs calibrées de $\mathrm{Ta}_{2} \mathrm{O}_{5}$ sont élaborées à $21{ }^{\circ} \mathrm{C}$ par croissance potentiostatique des films anodiques sur une plaque de tantale $\left(1 \mathrm{~cm}^{2}\right)$ plongée 2 heures dans une solution de $\mathrm{H}_{3} \mathrm{PO}_{4}$ 0,0016 M. Dans ces conditions [5], l'épaisseur $Z$ (nm) des films évolue linéairement en fonction du potentiel appliquée $E(\mathrm{~V})$ selon la relation :

$$
Z=1,896(E+1,9) .
$$

Certaines multicouches $\mathrm{Cu}-\mathrm{Co}$ ont été préparées par pulvérisation cathodique $\mathrm{RF}$ sous pression d'argon $\left(2,5\right.$ et $5 \times 10^{-2}$ mbar respectivement pour les échantillons dénommés $\mathrm{A}$ et $\left.\mathrm{B}\right)$ sur un substrat de verre préalablement recouvert d'un "buffer" de $10 \mathrm{~nm}$ de fer [16]. L'analyse de ces matériaux entrait dans le cadre des études proposées par le GDR 1108 - "caractérisation des interfaces des multimatériaux" [16]. Les épaisseurs des trois couches successives de cuivre, cobalt puis cuivre (ou inversement) proposées pour 30, 50 et $100 \mathrm{~nm}$ à l'élaboration ont été précisées par des analyses à la microsonde électronique quantifiée par le logiciel Strata [16]. D'autres furent élaborées par électrodéposition sous potentiel pulsé à partir d'un électrolyte unique contenant des sels de cobalt et de cuivre [17]. La détermination des rendements faradiques permet de connaître approximativement l'épaisseur des couches déposées sur des substrats de cuivre ou de verre conducteur ITO (Indium Tin Oxide).

\section{Approche quantitative de l'analyse en profondeur des couches $\mathrm{Cu}-\mathrm{Co}$}

\subsection{Calibrage des vitesses d'érosion}

Le calibrage du dispositif de profilométrie est réalisé à l'aide des couches d'oxyde de tantale préparées au laboratoire et érodées dans les conditions présentées précédemment. Rappelons les avantages de ces couches qui leur ont valu d'être choisies comme matériau de référence : mise en œuvre simple à l'aide d'un matériel peu onéreux, épaisseurs des films reproductibles [4] mais aussi interfaces abruptes et résolution en profondeur $\Delta Z$ inégalée qui peut être inférieure à $2 \mathrm{~nm}$ dans des conditions d'érosion optimisées d'incidence et d'énergie des ions $[15,18]$. 

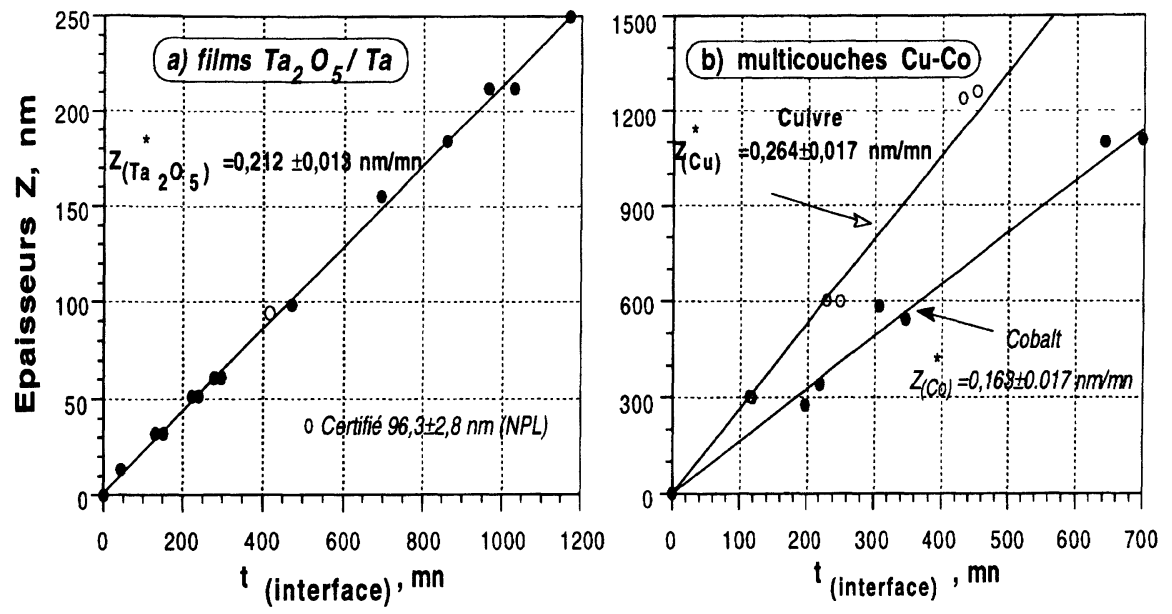

Fig. 1. - Calibrage des vitesses d'érosion : a) dans des couches d'épaisseurs connues d'oxyde de tantale, b) dans les couches de cuivre et cobalt du GDR.

[Sputter rates calibration: a) in $\mathrm{Ta}_{2} \mathrm{O}_{5}$ layers, b) in Co and Cu layers of known thicknesses.]

L'amorphisation de la couche d'oxyde et la grande différence des masses atomiques entre l'oxygène et le tantale limitent l'implantation de l'oxygène dans le substrat de tantale et expliquent ces bonnes performances en accord avec les prévisions théoriques $[18,19]$.

Les temps d'érosion nécessaires pour atteindre l'interface $\mathrm{Ta}_{2} \mathrm{O}_{5} /$ Ta sont reportés en fonction de l'épaisseur des films donnée par la relation (1) sur la figure 1a. Selon la définition ASTM, ce temps requis pour atteindre une intensité égale à $50 \%$ de celle des plateaux observés dans le film néglige complètement la contribution de la couche externe de pollution ; à cela s'ajoute la dissymétrie fréquente des interfaces qui rend cette définition peu réaliste et les mesures d'épaisseurs d'autant plus inexactes que les films sont plus minces. Aussi avons-nous décidé de déduire de la mesure de $t_{50 \%}$ la contribution de la couche de pollution que nous estimerons équivalente au temps d'érosion nécessaire pour que l'intensité de la transition du carbone décroisse de $50 \%$ de son intensité initiale.

Dans nos conditions expérimentales d'érosion, une vitesse d'érosion de $0,212 \pm 0,013 \mathrm{~nm} / \mathrm{mn}$ dans l'oxyde de tantale est ainsi obtenue par la mesure de la pente de la droite de régression représentant l'évolution de l'épaisseur des films en fonction des temps d'érosion $t_{50 \%}$ (Fig. 1a). Ce résultat est confirmé par l'analyse en profondeur d'un film d'épaisseur certifiée de $96,3 \pm$ $0,28 \mathrm{~nm}[20]$. Des vitesses d'érosion de 0,264 $\pm 0,017 \mathrm{~nm} / \mathrm{min}$ et de $0,163 \pm 0,017 \mathrm{~nm} / \mathrm{min}$ sont respectivement obtenues dans des couches de cuivre et de cobalt purs calibrées par microsonde électronique [16] et érodées dans ces mêmes conditions (Fig. 1b).

\subsection{Conversion des temps d'érosion en profondeurs érodées}

Le passage des différentes interfaces cuivre-cobalt se traduit par des variations brutales et successives de la composition et donc de la vitesse d'érosion. On définit alors une vitesse instantanée d'érosion $Z(t)^{*}$ reliée aux fractions atomiques instantanées $\mathrm{X}_{\mathrm{Cu}}(t)$ et $\mathrm{X}_{\mathrm{Co}}(t)$ (avec $\left.\mathrm{X}_{\mathrm{Cu}}(t)+\mathrm{X}_{\mathrm{Co}}(t)=1\right)$ et aux vitesses d'érosion $Z_{\mathrm{Co}}^{*}$ et $Z_{\mathrm{Cu}}^{*}$ dans les métaux purs Co et Cu par la relation :

$$
Z(t)^{*}=Z_{\mathrm{Cu}}^{*} \mathrm{X}_{\mathrm{Cu}}(t)+Z_{\mathrm{Co}}^{*} \mathrm{X}_{\mathrm{Co}}(t)
$$



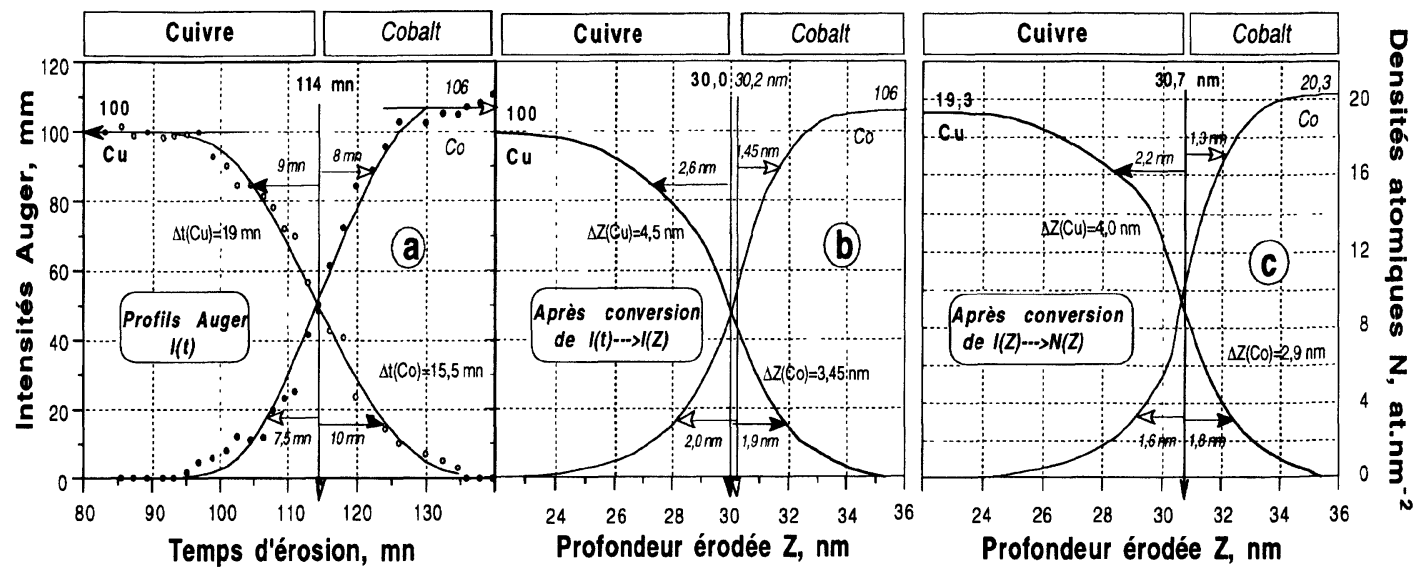

Fig. 2. - Quantification de l'analyse en profondeur des multicouches $\mathrm{Cu}-\mathrm{Co}$ (première interface $\mathrm{Cu}-\mathrm{Co}$ de l'échantillon 3A). a) Profils Auger $I(t)$; b) conversion des temps d'érosion en profondeurs érodées : profils $I(Z)$, c) conversion des intensités Auger en densités atomiques : profils $N(Z)$.

[Depth profile quantification of the first $\mathrm{Cu}$-Co interface (3A sample): a) Auger profiles $I=f(t)$, b) Sputter time conversion into eroded depth, $I(Z)$ profiles and c) Auger intensites conversion into atomic densities, $N(Z)$ concentration profiles.]

Les fractions atomiques $\mathrm{X}_{\mathrm{A}}(t)$, où $\mathrm{A}=\mathrm{Co}$ ou $\mathrm{Cu}$, ne sont pas connues à ce stade de la quantification, elle seront en première approximation assimilées aux rapports d'intensités $I_{\mathrm{Cu}} / I_{\mathrm{Cu}^{0}}$ et $I_{\mathrm{Co}} / I_{\mathrm{Co}^{\circ}}$, l'indice 0 se réfèrant au métal pur, et corrigées de l'effet de la profondeur d'échappement $\lambda$ des électrons Auger selon l'équation $[3,21]$ :

$$
\mathrm{X}_{\mathrm{A}}(t)=\left[I_{\mathrm{A}} / I_{\mathrm{A}^{0}}\right](t)-\lambda_{\mathrm{A}} \mathrm{d}\left[I_{\mathrm{A}} / I_{\mathrm{A}^{0}}\right](t) / Z(t)^{*} \mathrm{~d} t .
$$

La combinaison des équations (2) et (3) conduit à devoir résoudre une équation du second degré en $Z(t)^{*}$ pour chaque séquence d'érosion $\Delta t$ choisie suffisamment courte à l'interface pour être assimilable à $\mathrm{d} t$ dans l'équation (3). $\lambda_{\mathrm{A}}$ étant aussi censé évoluer avec la composition, nous utiliserons pour simplifier la résolution de la relation (3) une valeur numérique de $\lambda_{\mathrm{A}}$ moyennée entre celles calculées pour le matériau A pur et celle pour l'élément A infiniment dilué dans l'autre matériau $\mathrm{B}$ [22]. La conversion non-linéaire des temps d'érosion en profondeurs érodées s'effectue ensuite en sommant les $\Delta Z$ successifs calculés par le produit $Z(t)^{*} \Delta t$ (Figs. 2a et $2 \mathrm{~b})$. Pour réaliser ces conversions dans de bonnes conditions, les profils Auger expérimentaux sont préalablement lissés.

\subsection{Conversion des intensités Auger en concentrations}

Le calibrage des intensités Auger s'effectue en déterminant des facteurs de sensibilité déduits de l'analyse d'étalons constitués par les métaux purs, et en appliquant un modèle d'abrasion couche par couche qui, rappelons-le, n'est pas censé conduire au profil de concentration d'origine, c'est-à-dire non modifié par le bombardement d'ions, mais prend seulement en compte les effets de la profondeur d'échappement des électrons Auger [12,13]. Les effets de rétrodiffusion sont considérés comme étant du second ordre par rapport à ceux des profondeurs d'échappement ; ils sont ici d'autant plus négligeables qu'il s'agit d'éléments de numéros atomiques voisins [2]. 


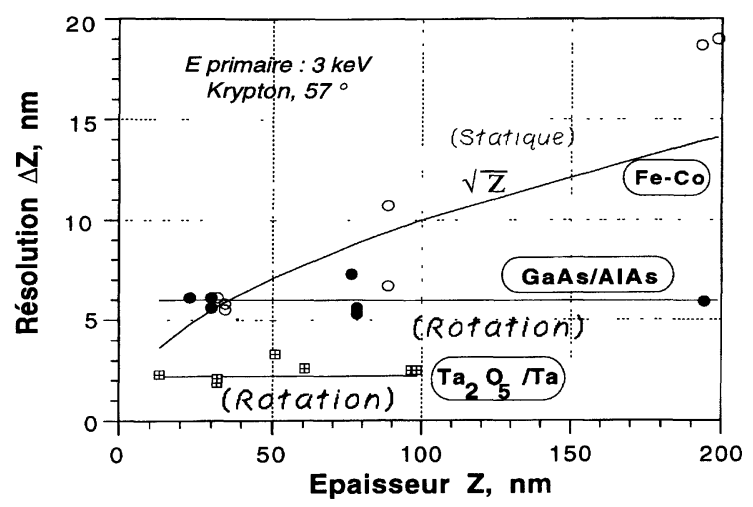

Fig. 3. - Calibrage de la résolution en profondeur dans les systèmes $\mathrm{Fe}-\mathrm{Co}$, AsGa-AlAs et $\mathrm{Ta}_{2} \mathrm{O}_{5}-\mathrm{Ta}$.

[Calibration of the depth resolution with the $\mathrm{Fe}-\mathrm{Co}, \mathrm{AsGa}-\mathrm{AlAs}$ and $\mathrm{Ta}_{2} \mathrm{O}_{5}-\mathrm{Ta}$ systems.]

Les concentrations atomiques sont volontairement exprimées en densités atomiques, et non en fractions atomiques ainsi que pourrait le fournir rapidement la relation (3), afin de pouvoir établir des bilans atomiques significatifs et comparatifs d'un profil à l'autre au terme de notre approche quantitative. Les densités atomiques $N_{\mathrm{A}}$ (en at $\left./ \mathrm{nm}^{2}\right)$ relatives à l'élément A se déduisent des intensités Auger $I_{\mathrm{A}}^{(\imath)}$ et $I_{\mathrm{A}}^{(i+1)}$ mesurées à des profondeurs différant de l'épaisseur moyenne $d(Z)$ d'une couche atomique par l'expression [12] :

$$
N_{\mathrm{A}}=\left[I_{\mathrm{A}}^{(i)}-k_{\mathrm{A}} I_{\mathrm{A}}^{(i+1)}\right] / \beta_{\mathrm{A}} .
$$

$k_{\mathrm{A}}$ et $\beta_{\mathrm{A}}$ sont respectivement les coefficients d'atténuation et de sensibilité relatifs aux électrons Auger de la transition considérée de l'élément A :

$$
\begin{aligned}
k_{\mathrm{A}} & =\exp \left[-d(Z) / \lambda_{\mathrm{A}}\right] \\
\text { et } \beta_{\mathrm{A}} & =I_{\mathrm{A}^{0}}\left(1-k_{\mathrm{A}}\right) / N_{\mathrm{A}^{0}} .
\end{aligned}
$$

Une seconde conversion de l'échelle des profondeurs $Z$ en nombre équivalent de couches atomiques devient alors nécessaire. L'évolution de l'épaisseur moyenne d'une couche atomique $d(Z)$ avec la composition est formulée de la même manière que la vitesse d'érosion en remplaçant $Z(t)^{*}, Z_{\mathrm{Cu}}^{*}$ et $Z_{\mathrm{Co}}^{*}$ respectivement par $d(Z), d_{\mathrm{Cu}}$ et $d_{\mathrm{Co}}$ dans la relation $(2)$ et en exprimant la relation (3) en fonction des profondeurs érodées. Dans le système $\mathrm{Cu}-\mathrm{Co}$, cette correction de $d(Z)$ avec la composition est mineure car $d(Z)$ ne varie qu'entre $d_{\mathrm{Co}}=0,222 \mathrm{~nm}$ et $d_{\mathrm{Cu}}=0,228 \mathrm{~nm}$. La dépendance du coefficient d'atténuation $k_{\mathrm{A}}$ et des libres parcours moyens inélastiques $\lambda_{i \mathrm{~A}}=\lambda_{\mathrm{A}}(\cos \theta)^{-1}$ en fonction de $d(Z)$, en $\mathrm{nm}$, s'exprime grâce à la formulation Seah et al. [22] selon :

$$
k_{\mathrm{A}}(Z)=\exp \left[-1 / 0,41 d(Z)^{0,5} E_{\mathrm{A}}^{0,5} \cos \theta\right]
$$

où $E_{\mathrm{A}}$ est l'énergie cinétique $(\mathrm{eV})$ des électrons Auger de la transition retenue pour l'élément $\mathrm{A}$ et $\theta$ l'angle moyen de collection de l'analyseur pour une incidence de $30^{\circ}$ du faisceau d'électrons $(\cos \theta=0,64)$.

Après une dernière conversion des abscisses en profondeurs érodées $Z$, les profils de concentration $N[Z]$ obtenus vont permettre tout à la fois de réaliser une mesure quantitative de la résolution en profondeur aux interfaces et de l'épaisseur des couches selon les normes ASTM ou d'après les bilans atomiques établis dans les différentes couches. 


\subsection{Calibrage de la résolution en profondeur $\Delta Z$}

Dans nos conditions d'abrasion (ions krypton, $3 \mathrm{keV}, 57^{\circ}$ d'incidence, rotation Zalar), la résolution en profondeur aux interfaces $\mathrm{Ta}_{2} \mathrm{O}_{5} / \mathrm{Ta}$ est de 2,5 $\pm 1,0 \mathrm{~nm}$ et reste sensiblement indépendante de l'enterrement des interfaces. Notons que la détermination de $\Delta Z$ n'est pas ici entièrement rigoureuse car la conversion de $\Delta t$ en $\Delta Z$ s'effectue en supposant la vitesse d'érosion dans $\mathrm{Ta}_{2} \mathrm{O}_{5}$ identique à celle dans le tantale. Optimiser les conditions d'abrasion (angle d'incidence, énergie des ions, nature des ions, etc.) et le traitement du substrat pour éliminer l'oxyde natif avant l'anodisation sont de première importance pour espérer accéder à de très hautes résolutions comme celles reportées dans le système $\mathrm{Ta}_{2} \mathrm{O}_{5} / \mathrm{Ta}$ par Seah et al . (1,5 et $1,6 \mathrm{~nm}$ à 30 et $100 \mathrm{~nm}$ de profondeur) [18].

Lorsqu'il s'agit d'interfaces semi-conducteur/semi-conducteur (ou métal/métal), $\Delta Z$ atteint des valeurs plus élevées avec $6 \pm 1 \mathrm{~nm}$ mais reste constante en fonction de la profondeur (AsGa/AlAs, Fig. 3). Par contre, en mode "statique", la résolution en profondeur évolue sensiblement selon les prévisions avec la profondeur en fonction de $Z^{1 / 2}$ (couches $\mathrm{Fe}-\mathrm{Co}$, Fig. 3). La mise en œuvre d'un porte-échantillons tournant confirme le rôle déterminant de cet accessoire pour limiter l'élargissement des interfaces par un développement de la rugosité induite par un bombardement d'ions unidirectionnel.

\section{Résultats et discussion}

Deux paires d'échantillons multicouches [3A, 3B : $\mathrm{Cu}(30 \mathrm{~nm}) / \mathrm{Co}(50 \mathrm{~nm}) / \mathrm{Cu}(100 \mathrm{~nm}) /$ $\mathrm{Fe}(10 \mathrm{~nm})$ et $4 \mathrm{~A}, 4 \mathrm{~B}: \mathrm{Co}(30 \mathrm{~nm}) / \mathrm{Cu}(50 \mathrm{~nm}) / \mathrm{Co}(100 \mathrm{~nm}) / \mathrm{Fe}(10 \mathrm{~nm})]$ élaborées par pulvérisation cathodique et des échantillons comportant 3 bicouches $\mathrm{Cu}-\mathrm{Co}(6 \times 20 \mathrm{~nm})$ électrodéposées ont été analysés dans cette étude par profilométrie Auger.

La figure 4a représente les profils Auger réalisés sur un échantillon (3A) préparé par pulvérisation cathodique : les couches et interfaces analysées sont pures si on excepte la couche externe de pollution et la couche interne consistant en $10 \mathrm{~nm}$ de fer partiellement oxydé au contact du substrat de verre et l'échantillon $4 \mathrm{~B}$ qui présente une teneur résiduelle importante en oxygène dans toutes les couches, ce qui limitera la quantification de son analyse en profondeur (aucune épaisseur de couche ne sera proposée pour cet échantillon dans le tableau I, $\Delta Z$ sera estimée en prenant une valeur moyenne des vitesses d'érosion). Les couches électrodéposées $\mathrm{Cu} / \mathrm{Co}$ ne sont pas bien résolues malgré des épaisseurs choisies volontairement importantes d'environ $20 \mathrm{~nm}$ (Fig. 4b) ; elles sont moins pures que les précédentes et contiennent des teneurs notables en oxygène sur toute l'épaisseur du film. Le "buffer" de cuivre déposé sur le substrat ITO est presque inexistant, il semble s'être combiné majoritairement à l'oxygène du substrat ITO rendant l'interface avec le substrat particulièrement diffuse.

L'analyse en profondeur plus détaillée des multicouches préparées par pulvérisation cathodique permet de préciser l'apport de la quantification à la caractérisation de ces interfaces. Les corrections successives de vitesses d'érosion et de profondeurs d'échappement apparaissent plus clairement en considérant les résolutions relatives $\Delta t / t$ mesurées sur les profils Auger $I(t)$ et $\Delta Z / Z$ déterminées sur les profils $I(Z)$ puis $N(Z)$. Ainsi, dans le cas de la première interface peu déformée de échantillon $3 \mathrm{~A}$, la résolution relative sur le profil du cuivre s'améliore de 0,167 à 0,150 après correction des vitesses d'érosion (Figs. $2 \mathrm{a}$ et $2 \mathrm{~b}$ ) puis de 0,150 à 0,130 après celle des profondeurs d'échappement (Figs. $2 \mathrm{~b}$ et 2c), soit globalement une amélioration de $28 \%$ de la résolution (environ 1,1 nm) grâce à la mise en œuvre de l'approche quantitative.

Il est intéressant de noter la dissymétrie existant de part et d'autre de l'interface sur les profils d'intensité $I(Z)$ et de concentration $N(Z)$ qui n'apparaissait pas au premier abord sur les profils Auger $I(t)$ (Figs. 2a, 2b et 2c) : la partie la moins bien résolue des profils est 

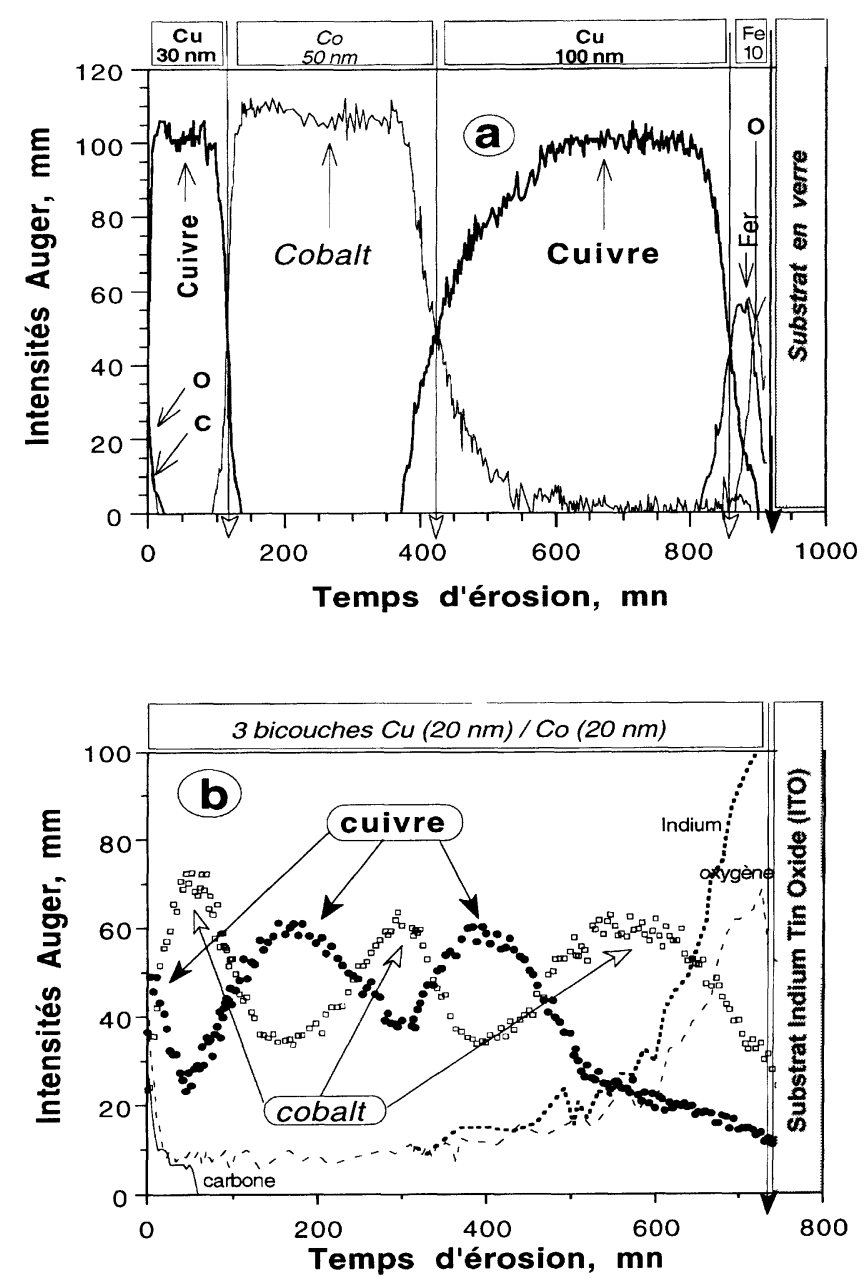

Fig. 4. - Profils Auger réalisés sur : a) un échantillon (3A) élaboré par pulvérisation cathodique et b) sur un échantillon préparé par électrodéposition.

[Auger sputter profiles performed on: a) a RF sputter deposited sample (3A) and b) on an electrodeposited sample.]

toujours située du côté de la matrice de cuivre quelle que soit l'interface considérée $(\mathrm{Co}-\mathrm{Cu}$ ou $\mathrm{Fe}-\mathrm{Cu}$ ). Des rugosités induites par le bombardement d'ions, différentes dans les deux métaux et subsistant à l'état résiduel en rotation Zalar, pourraient être à l'origine de cette dissymétrie. En effet, les couches de cuivre érodées de cette manière deviennent beaucoup plus rugueuses que celles de cobalt, telles sont les conclusions des examens pratiqués par microscopie à force atomique (AFM) sur des biseaux très inclinés obtenus par déplacement d'un échantillon identique en cours d'analyse SIMS [23].

Les interfaces relatives au dépôt de cobalt sur cuivre $(\mathrm{Co} \rightarrow \mathrm{Cu})$ apparaissent largement déformées et présentent des trainées importantes de cobalt dans la couche de cuivre conduisant à des résolutions de l'ordre de 15 à $30 \mathrm{~nm}$ assez inattendues en rotation Zalar (Fig. 6, seconde interface). La dissymétrie prononcée de ces interfaces pose évidemment la question de l'applicabilité 
Table I. - Épaisseurs des couches de cobalt ou de cuivre mesurées selon la norme ASTM (déduction faite de la couche de pollution externe pour la mesure de la première couche) ou déduite des bilans atomiques BA (en supposant les couches pures et denses) et résolution en profondeur mesurée sur les profils de concentration du cuivre aux différentes interfaces Cu/Co ou $\mathrm{Cu}, \mathrm{Co} / \mathrm{Fe}$ des échantillons préparés par pulvérisation cathodique.

[Cobalt and copper layer thicknesses according to ASTM measurements (with the superficial pollution layer deducted from the first layer thickness determination) or deduced from atomic balances BA (assuming pure and dense layers) and depth resolution measured on the copper concentration profile at the successive interfaces of the $\mathrm{RF}$ sputtered $\mathrm{Cu}-\mathrm{Co}$ samples.]

\begin{tabular}{|c|c|c|c|c|c|c|c|c|}
\hline & \multicolumn{3}{|c|}{$\begin{array}{c}\text { Couche Interface } \\
\mathrm{N}^{\circ} 1\end{array}$} & \multicolumn{3}{|c|}{$\begin{array}{c}\text { Couche Interface } \\
\mathrm{N}^{\circ} 2\end{array}$} & \multicolumn{2}{|c|}{$\begin{array}{c}\text { Couche } \\
\mathrm{N}^{\circ} 3\end{array}$} \\
\hline & \multicolumn{2}{|c|}{$\begin{array}{l}\text { Épaisseur } \\
(\mathrm{nm})\end{array}$} & \multirow{2}{*}{$\begin{array}{c}\Delta Z(\mathrm{Cu}) \\
(\mathrm{nm})\end{array}$} & \multirow{2}{*}{\multicolumn{2}{|c|}{$\begin{array}{c}\text { Épaisseur } \\
(\mathrm{nm}) \\
\text { ASTM BA }\end{array}$}} & \multirow[t]{2}{*}{$\begin{array}{c}\Delta Z(\mathrm{Cu}) \\
(\mathrm{nm})\end{array}$} & $\begin{array}{l}\text { Épaisseur } \\
(\mathrm{nm})\end{array}$ & \multirow{2}{*}{$\begin{array}{c}\Delta Z \\
(\mathrm{~nm}) \\
\mathrm{Cu}, \mathrm{Co} / \mathrm{Fe}\end{array}$} \\
\hline & ASTM & BA & & & & & ASTM BA & \\
\hline $3 \mathrm{~A}-\mathrm{Cu} / \mathrm{Co} / \mathrm{Cu} / \mathrm{Fe}$ & 29,1 & 29,1 & 4,0 & 50,8 & 55,2 & 33,0 & $109,0 \quad 103,2$ & 10,6 \\
\hline $3 \mathrm{~B}-\mathrm{Cu} / \mathrm{Co} / \mathrm{Cu} / \mathrm{Fe}$ & 29,6 & 29,6 & 4,6 & 58,2 & 61,8 & 30,3 & $114,0 \quad 109,1$ & 9,3 \\
\hline $\begin{array}{c}4 \mathrm{~A}-\mathrm{Co} / \mathrm{Cu} / \mathrm{Co} / \mathrm{Fe} \\
\text { (statique) }\end{array}$ & $\begin{array}{l}35,7 \\
35,8\end{array}$ & $\begin{array}{l}39,8 \\
39,9)\end{array}$ & $\begin{array}{c}20,0 \\
(22,2)\end{array}$ & $\begin{array}{r}61,0 \\
(65,4\end{array}$ & $\begin{array}{l}57,7 \\
68,8)\end{array}$ & $\begin{array}{c}10,7 \\
(27,0)\end{array}$ & $\begin{array}{cl}107,0 & 107,3 \\
(101,2 & 116,2)\end{array}$ & $\begin{array}{r}7,5 \\
(26,0)\end{array}$ \\
\hline $4 \mathrm{~B}-\mathrm{Co} / \mathrm{Cu} / \mathrm{Co} / \mathrm{Fe}$ & $\ldots$ & $-\ldots$ & 11,5 & $-\cdots$ & $-\ldots$ & 7,8 & $\ldots$ & 6,5 \\
\hline
\end{tabular}

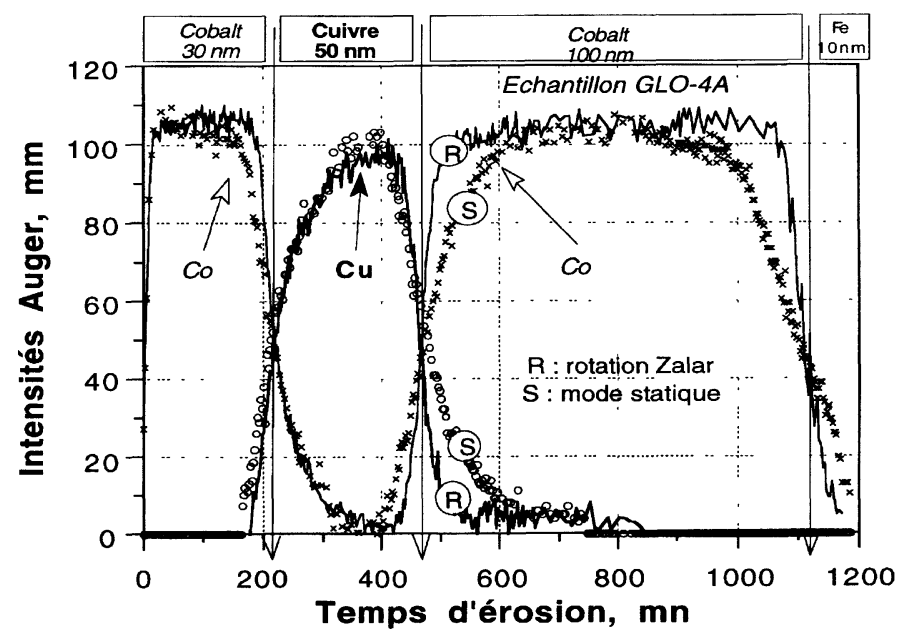

Fig. 5. - Profils Auger relatifs à l'échantillon 4A analysé en mode statique (S) et en rotation Zalar (R).

[AES Depth profile analysis of the RF sputter deposited $\mathrm{Cu}$-Co multilayer sample achieved in the usual static mode (S) and according to the Zalar rotation method (R).]

des normes ASTM à la mesure de l'épaisseur de ce genre de couches. L'établissement de bilans atomiques sur les profils de concentration offre par contre la possibilité de caractériser l'épaisseur des couches par des quantités d'atomes par unité de surface qui présentent alors l'intérêt de pouvoir être comparées directement à celles proposées par d'autres techniques analytiques telles que la rétrodiffusion d'ions RBS [16]. Nous avons reporté dans le tableau I les épaisseurs de couches mesurées selon les normes ASTM ou déduites des bilans atomiques 


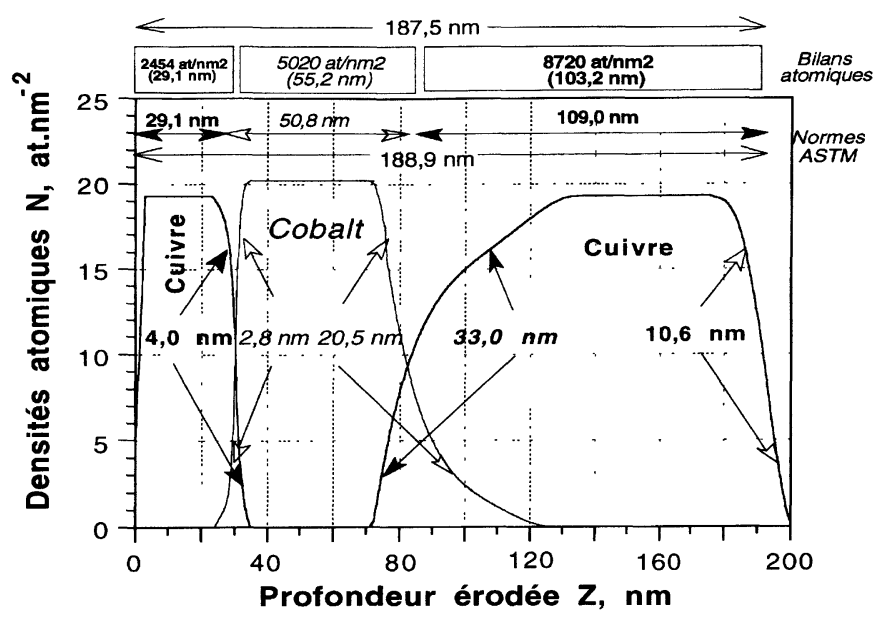

Fig. 6. - Profils de concentration et résolution en profondeur dans l'échantillon multicouches $\mathrm{Cu}-\mathrm{Co}$ (3A) élaboré par pulvérisation cathodique aqueuse.

[Depth concentration profiles and depth resolution in the $\mathrm{Cu}-\mathrm{Co}$ multilayer sample elaborated by $\mathrm{RF}$ sputter deposition (3A).]

en supposant une densité théorique des films analysés (BA). Celles-ci apparaissent sensiblement équivalentes pour les premières couches étudiées à condition de déduire, comme ici, l'épaisseur de la couche de pollution. Par ailleurs, les épaisseurs ASTM des couches de cobalt apparaissent sous-estimées par rapport à la mesure BA et, inversement, celles des couches de cuivre surestimées quand intervient dans la mesure une interface $\mathrm{Co} \rightarrow \mathrm{Cu}$ fortement déformée (Tab. I : première couche de cobalt des échantillons 4A et 4B et seconde couche des échantillons $3 \mathrm{~A}$ et $3 \mathrm{~B})$. Ces considérations expliquent les divergences pouvant se manifester à propos des mesures d'épaisseur de couches réalisées sur des échantillons identiques mais par des techniques analytiques différentes qui se réfèrent à l'une ou l'autre des définitions pour qualifier les épaisseurs [16].

Ainsi donc, la résolution en profondeur aux différentes interfaces évolue aléatoirement en fonction de la profondeur (Fig. 6), elle paraît même s'améliorer dans certains échantillons (4A et $4 \mathrm{~B}$, Tab. I) mais en aucun cas elle ne demeure constante comme cela pouvait être envisagé en rotation Zalar. En fait, dans les quatre échantillons étudiés, il faut faire la distinction entre les interfaces $\mathrm{Co} \rightarrow \mathrm{Cu}$ et les autres $(\mathrm{Cu} \rightarrow \mathrm{Co}, \mathrm{Co} \rightarrow \mathrm{Fe}$ ou $\mathrm{Cu} \rightarrow \mathrm{Fe})$. Les premières présentent toutes des résolutions anormalement élévées comprises entre 12 et $33 \mathrm{~nm}$. Inversement, la résolution aux interfaces $\mathrm{Cu} \rightarrow \mathrm{Co}$ et $(\mathrm{Cu}$ ou $\mathrm{Co}) \rightarrow$ Fe reste comprise entre 7 et $11 \mathrm{~nm}$ aux profondeurs supérieures à $30 \mathrm{~nm}$, profondeur jugée nécessaire pour que s'instaure l'état stationnaire de la pulvérisation ionique. Dans nos conditions d'érosion non optimisées (incidence et énergie des ions), ces valeurs paraissent grossièrement représentatives, si on écarte les différences de rugosité résiduelle évoquées plus haut, de la contribution du mixage ionique à l'élargissement d'interfaces métal/métal supposées initialement abruptes. Cette contribution est en accord avec les mesures $\left(6<\Delta Z<10 \mathrm{~nm}, \mathrm{Ar}^{+}, 3 \mathrm{keV}, 45^{\circ}\right)$ faites dans des conditions voisines sur des couples métal/métal en rotation par Zalar et al. [24] ; elle est, comme prévu, plus élevée qu'à des interfaces semi-conducteur/semi-conducteur $(\Delta Z=6 \pm 1 \mathrm{~nm})$, métal/oxyde $(\Delta Z=2,5 \pm 1 \mathrm{~nm}$, Fig. 3) ou oxyde/oxyde [24].

L'effet bénéfique de la rotation Zalar se traduit sans ambiguïté sur les profils Auger réalisés avec (R) ou sans (S) rotation par un élargissement bien moindre des interfaces (Fig. 5). 
À la première interface $\mathrm{Co} \rightarrow \mathrm{Cu}$ de cet échantillon, située à $30 \mathrm{~nm}$, la résolution en rotation $\Delta Z_{[\mathrm{R}]}=20,0 \mathrm{~nm}$, anormalement élevée à cette profondeur, demeure néanmoins proche de celle observée en mode statique $\Delta Z_{[\mathrm{S}]}=22,2 \mathrm{~nm}$. Aux interfaces plus profondes et qualifiées d'abruptes $(\mathrm{Cu} \rightarrow \mathrm{Co}$ et $\mathrm{Co} \rightarrow \mathrm{Fe})$, la résolution en profondeur $\Delta Z$ décroît par contre significativement d'environ $25 \mathrm{~nm}$ en mode statique $(\mathrm{S})$ à $8-10 \mathrm{~nm}$ en rotation (R) (Tab. I). Ces observations permettent de conclure que, si la rotation Zalar peut limiter la dégradation de la résolution en profondeur aux interfaces initialement abruptes, elle ne peut corriger les interfaces d'une rugosité importante préexistante comme cela semble apparaître aux interfaces $\mathrm{Co} \rightarrow \mathrm{Cu}$. On peut donc imaginer une déformation de ces interfaces se produisant avant l'analyse en profondeur lors du dépôt de cobalt sur la couche de cuivre. En effet, il semblerait irréaliste de vouloir attribuer de telles pertes de résolution $(\Delta Z>15 \mathrm{~nm})$, aux énergies primaires mises en jeu, au processus de mélange collisionnel d'atomes, qu'il soit décrit par un modèle "balistique" $[19,25,26]$ ou par un modèle de diffusion accélérée sous irradiation [27]. Il en est de même pour le modèle des pointes thermiques proposé par Vineyard ("thermal spike model") [28] qui fut appliqué en premier lieu au mixage d'atomes par des ions de hautes énergies $[29,30]$ puis par des ions de quelques $\mathrm{keV}$ d'énergie comme en analyse de surfaces [14,31]. Ce modèle arrive en effet à expliquer qualitativement les différences de résolution en profondeur observées en fonction des caractéristiques thermodynamiques (enthalpies de mélange et de cohésion) des couples d'éléments en présence aux interfaces mais pas celles observées aux interfaces $\mathrm{Co} \rightarrow \mathrm{Cu}$ et $\mathrm{Co} \rightarrow \mathrm{Fe}$ ou $\mathrm{Cu} \rightarrow \mathrm{Co}$. Selon ce modèle, les systèmes à enthalpies de mélange $\Delta H_{\mathrm{m}}$ fortement exothermiques favorisent sous bombardement d'ions la mobilité atomique aux interfaces et la dégradation de la résolution [31]. Les systèmes $\mathrm{Pt}-\mathrm{Ti}$ et $\mathrm{Pt}-\mathrm{Ni}$ aux enthalpies de mélange très différentes $\left(\Delta H_{\mathrm{m}}=-122\right.$ et $\left.-7 \mathrm{~kJ} / \mathrm{mole}\right)$ ont été choisis à cet effet par Cirlin et al. [14] pour tester le modèle et montrer que la contribution du mixage ionique à ces deux types d'interfaces évoluait selon les prédictions (6 et $4 \mathrm{~nm}$ respectivement). Pour les systèmes $\mathrm{Cu}-\mathrm{Co}$ et $\mathrm{Co}-\mathrm{Fe}[32]$, des enthalpies respectives de mélange légèrement positive de $6 \mathrm{~kJ} /$ mole ou faiblement négative $-1 \mathrm{~kJ} /$ mole laissent donc envisager des interfaces étroites et bien résolues ; des enthalpies de dissolution en solution infiniment diluée $\mathrm{Cu}$ dans Co ou de Co dans $\mathrm{Cu}$, pratiquement identiques $(+26$ et $+25 \mathrm{~kJ} / \mathrm{mole}$ ), semblent aussi jouer en faveur d'un comportement équivalent des métaux aux deux types d'interfaces $\mathrm{Cu} \rightarrow \mathrm{Co}$ ou $\mathrm{Co} \rightarrow \mathrm{Cu}[32]$. Ces considérations prédictives sur l'effet du mixage ionique d'atomes aux interfaces du couple $\mathrm{Cu}-\mathrm{Co}$ ne permettent donc pas d'interpréter les anomalies observées aux interfaces spécifiques $\mathrm{Co} \rightarrow \mathrm{Cu}$.

Par contre, les nombreuses études réalisées ces dernières années sur la préparation de multicouches $\mathrm{Cu}-\mathrm{Co}$ comme matériau candidat à la magnétorésistance géante ont mis en évidence certains effets au cours de la phase de croissance de la couche de cobalt sur le cuivre. Une controverse existe actuellement sur les mécanismes de croissance proposés, couche par couche [33] et par îlots tridimensionnels [11,34], pour ces dépôts de cobalt sur du cuivre. Selon Bauer [35], une différence positive entre les énergies de surface de l'élément du film et de celui du substrat favorise une croissance par îlots tridimensionnels (Volmer-Weber). Dans le système $\mathrm{Cu}-\mathrm{Co}$, les énergies de surface très différentes du cobalt $\left(2550 \mathrm{~mJ} / \mathrm{m}^{2}\right)$ et du cuivre $\left(1850 \mathrm{~mJ} / \mathrm{m}^{2}\right)$ laissent envisager ce type de croissance et même la ségrégation des atomes du substrat $(\mathrm{Cu})$ entre les îlots de cobalt [36]. Ces prédictions ont été vérifiées expérimentalement [11,34], les résultats obtenus montrent que les interfaces $\mathrm{Co} \rightarrow \mathrm{Cu}$ (et $\mathrm{Fe} \rightarrow \mathrm{Cu}$ ) sont instables dès la température ambiante [37]. Ces processus de diffusion superficielle du cuivre entre les îlots de cobalt ne sont mis en évidence qu'à faible vitesse de dépôt (environ 1 monocouche par minute) et sont bien entendu activés par la température de sorte qu'ils ne devraient pas se produire aux vitesses beaucoup plus rapides $(\times 100)$ utilisées pour l'élaboration de nos matériaux par pulvérisation cathodique à la température ambiante [11]. En conclusion, l'extension des queues de cobalt 


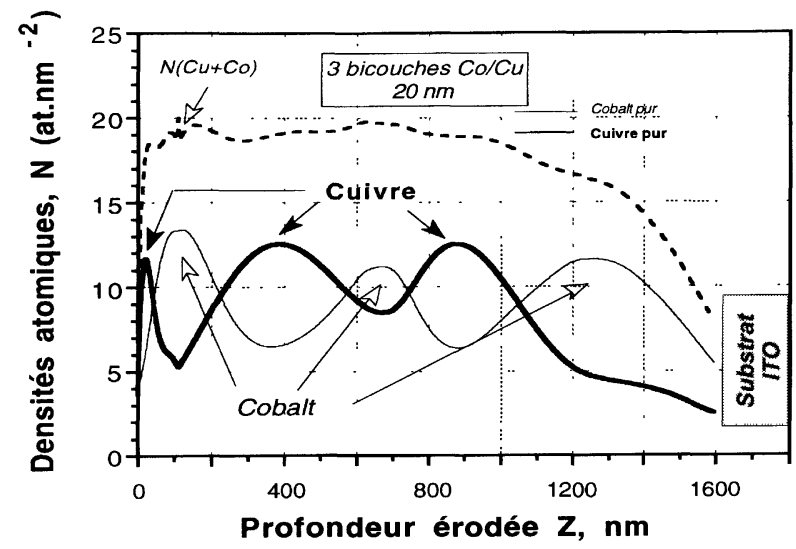

Fig. 7. - Profils de concentration dans un échantillon multicouche (3 bicouches de $20 \mathrm{~nm})$ préparé par électrodéposition.

[Depth concentration profiles in a $\mathrm{Cu}-$ Co electrodeposited multilayer sample ( 3 bilayers, $d \approx 20 \mathrm{~nm})$.]

dans les couches de cuivre restent inexpliquées à ce stade d'avancement des recherches ; des études complémentaires vont être entreprises pour élaborer des interfaces modèles $\mathrm{Co} \rightarrow \mathrm{Cu}$ in situ le système d'analyse nous permettant de mieux contrôler les étapes d'élaboration et d'analyse des échantillons.

Les profils de concentration relatifs aux couches $\mathrm{Cu}-\mathrm{Co}$ élaborées par électrodéposition (3 bicouches de $20 \mathrm{~nm}$ ) présentés dans la figure 7 sont semi-quantitatifs car l'influence de l'oxygène résiduel sur les vitesses d'érosion est difficilement quantifiable. Une disparité certaine existe entre l'épaisseur des diverses couches analysées ; de fait, l'épaisseur globale des 3 bicouches s'avère plus proche de $150 \mathrm{~nm}$ que des $120 \mathrm{~nm}$ annoncés. En particulier, la couche superficielle de cuivre apparaît beaucoup plus mince qu'annoncée et ceci sur tous les échantillons analysés ; il est en effet probable qu'une partie du cuivre déposé puisse se dissoudre lors de la coupure de courant mettant un terme à l'expérience. Une épaisseur de $20 \mathrm{~nm}$ pour chaque couche, jointe à une résolution en profondeur typiquement de $10 \mathrm{~nm}$, devrait cependant permettre la complète résolution de ces multicouches. Ce n'est pas le cas observé sur la figure 7 où la résolution est moins bonne que prévue, toutefois aucune déformation spécifique n'est mise en évidence au cours de ces analyses aux interfaces $\mathrm{Co} \rightarrow \mathrm{Cu}$. D'après Hofmann [38], la différence de concentrations $N_{\mathrm{m}}$ entre maxima et minima des ondulations de profils rapportés à la concentration $N^{0}$ dans l'élément pur sont reliées à l'épaisseur des couches $d$ et à $\Delta Z$ selon :

$$
N_{\mathrm{m}} / N^{0} \approx 1-2\left[\operatorname{erf}\left(3 d / 2^{1 / 2} \Delta Z\right)-\operatorname{erf}\left(d / 2^{1 / 2} \Delta Z\right)\right]
$$

Pour ces couches d'épaisseurs voisines de $20 \mathrm{~nm}$, une résolution en profondeur moyenne de $22 \mathrm{~nm}$ est dérivée des valeurs numériques de $N_{\mathrm{m}}$ par la relation (8). Comme dans les couches préparées par pulvérisation cathodique, des valeurs aussi élevées de $\Delta Z$ ne s'expliquent pas en rotation Zalar pour des interfaces supposées abruptes mais peuvent s'interpréter si l'électrodéposition n'est pas complètement sélective ou s'il s'opère une redissolution partielle au changement de potentiel lorsqu'on passe du dépôt de cobalt à celui de cuivre. Ici aussi des analyses complémentaires sont envisagées en particulier avec des films plus épais afin d'établir s'il est possible de résoudre les couches successives pour valider ou infirmer la sélectivité de la méthode d'électrodéposition mais aussi pour s'assurer définitivement que la présence des trainées de cobalt dans le cuivre est le fait d'une technique d'élaboration plutôt que celui de la pulvérisation ionique. 


\section{Conclusions}

L'analyse en profondeur de matériaux multicouches $\mathrm{Cu}-\mathrm{Co}$ est réalisée par spectrométrie d'électrons Auger, la quantification s'effectue grâce à un calibrage des vitesses d'abrasion ionique et des sensibilités élémentaires dans le cobalt et le cuivre purs et en pratiquant une correction des profondeurs d'échappement des électrons Auger. Cette procédure permet d'accéder à des profils de concentration, exprimés en densités atomiques, qui autorisent une mesure significative des épaisseurs de couches, de la résolution en profondeur aux interfaces et permet l'établissement de bilans atomiques comparatifs.

La rugosité de surface induite par le bombardement d'ions est minimisée par la rotation Zalar des échantillons en cours d'abrasion ; toutefois une rugosité résiduelle plus élevée dans le cuivre que dans le cobalt serait à l'origine de l'élargissement plus prononcé des profils dans la matrice de cuivre. Ainsi une résolution de 7 à $10 \mathrm{~nm}$ indépendante de la profondeur $(Z>30 \mathrm{~nm})$ est déterminée dans nos conditions expérimentales d'érosion aux interfaces dites "normales" $(\mathrm{Cu}$ déposé sur $\mathrm{Co}$ et $\mathrm{Cu}$ ou Co déposés sur $\mathrm{Fe}$ ) des échantillons élaborés par pulvérisation cathodique. Cette résolution peut être considérée comme grossièrement représentative du mixage ionique d'atomes s'opérant aux interfaces.

Les interfaces $\mathrm{Co} \rightarrow \mathrm{Cu}$ issues du dépôt de cobalt sur du cuivre présentent par contre un comportement qualifié d'anormal due à la présence de trainées de cobalt dans les couches de cuivre qui se traduit par une résolution en profondeur supérieure à $15 \mathrm{~nm}$. Ces anomalies flagrantes en rotation Zalar sont masquées en mode statique par une évolution monotone

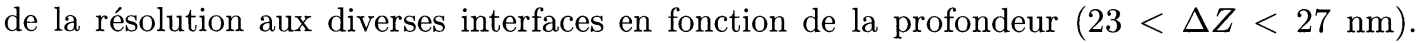
Cette méthode s'affirme donc indispensable pour affiner la caractérisation des interfaces lors de l'analyse en profondeur de ces matériaux, il lui est cependant impossible d'améliorer la résolution en profondeur d'interfaces initialement rugueuses comme cela semble être le cas avec les dépôts de cobalt sur cuivre. L'ordre de grandeur de l'élargissement de ces interfaces ne paraît pas compatible avec ceux prédits par les modèles "balistiques" ou thermodynamiques de pointes thermiques censés décrire le mixage ionique d'atomes. Ces anomalies paraissent donc imputables aux conditions d'élaboration de ces matériaux et, en particulier, à la difficulté à pouvoir maîtriser la préparation des interfaces $\mathrm{Co} \rightarrow \mathrm{Cu}$ dont l'instabilité, liée à la grande différence d'énergies de surface entre le cobalt et le cuivre, a été récemment mise en évidence. Cependant, les vitesses de dépôts pratiquées ici en pulvérisation cathodique semblent trop rapides $(\times 10-100)$ pour que le mode de croissance et le processus d'interdiffusion incriminés à ce propos soient succeptibles d'affecter les interfaces dès la température ambiante.

L'analyse en profondeur des multicouches électrodéposées $\mathrm{Cu}$-Co a permis de mettre en évidence un comportement atypique de ces interfaces $(\Delta Z \approx 22 \mathrm{~nm})$ compte-tenu de l'épaisseur prévue et de la périodicité des couches qui semble en relation avec la sélectivité de la méthode de dépôt.

\section{References}

[1] Norme ASTM. E 1438-91, november 1991, Annual Book of ASTM Standards, 1916 Race Street, Philadelphia, PA 19103 (USA).

[2] Hofmann S., "Practical Surface Analysis" (2nd edition), D. Briggs and M. P. Seah Eds. (Wiley, Chichester, 1990) Chap. 4.

[3] Hofmann S. et Zalar A., Thin Solid Films 60 (1979) 201. 
[4] Seah M.P., Mathieu H.J. et Hunt C.P., Surf. Sci. 139 (1984) 549.

[5] Seah M.P., Holburn M.W., Ortega C. et Davies J.A., Nucl. Instr. Meth. B 30 (1988) 128.

[6] Zalar A., Thin Solid Films 124 (1985) 123.

[7] Zalar A., Surf. Interface Anal. 9 (1986) 41.

[8] Hofmann S., J. Vac. Sci. Technol. A 9 (1991) 1466.

[9] Zalar A. et Hofmann S., Appl. Surf. Sci. 68 (1993) 361.

[10] Majkrzak C.F., Cable J.W., Kwo J., Hong M., Mc Whan D.B., Yafet Y., Waszczak J.V. et Vettier C., Phys. Rev. Lett. 56 (1986) 2700.

[11] Rabe A., Memmel N., Steltenpohl A. et Fauster Th., Phys. Rev. Lett. 73 (1994) 2728.

[12] Pons F, Le Héricy J. et Langeron J.-P., Surf. Sci. 69 (1977) 547.

[13] Langeron J.-P. et Lorang G., Analusis 17 (1989) 31.

[14] Cirlin E.-H., Cheng Y.-T., Ireland P. et Clemens B., Surf. Interface Anal. 15 (1990) 337.

[15] Hunt C.P. et Seah M.P., Surf. Interface Anal. 5 (1983) 199.

[16] Rapport final d'activité 1994-1997, Groupement de Recherche du CNRS GDR G1108 "Caractérisation des interfaces dans les multimatériaux", juin 1997.

[17] Nallet P., Chassaing E., Walls M.G. et Hÿtch M.J., J. Appl. Phys. 79 (1996) 6884 ; Nallet P., Thèse de Doctorat de l'Université Paris VI (décembre 1997).

[18] Hunt C.P. et Seah M.P., Surf. Interface Anal. 15 (1990) 254.

[19] Schiott H.E., Radiation Effects 6 (1970) 107.

[20] Matériau de référence $\mathrm{Ta}_{2} \mathrm{O}_{5}, \mathrm{BCR}$ n261, NPL nS7B83 (National Physical Laboratory, DMA, Teddington, Middlesex TW11 OLW-UK).

[21] Iwasaki H. et Nakamura G., Surf. Sci. 57 (1976) 779.

[22] Seah M.P. et Dench W.A., Surf. Interface Anal. 1 (1979) 1.

[23] Grattepain Ch., Dubois C., Repoux M., Ben Youssef O., Tromson-Carli A. et Le Duigou J., Colloque I2M "interfaces et multimatériaux", Aix-en-Provence, mai 1997.

[24] Zalar A., Panjan P., Krasevec V. et Hofmann S., Surf. Interface Anal. 19 (1992) 50.

[25] Sigmund P., Appl. Phys. Lett. 14 (1969) 114.

[26] Littmark U. et Hofer W.O., "Thin Film and Depth Profile Analysis", H. Oerschner Ed. (Springer -Verlag, Berlin, 1984) Chap. 8, p. 159.

[27] Seran J-L. et Limoge Y., Surf. Sci. 107 (1981) 176.

[28] Vineyard G.H., Radiat. Effects 29 (1976) 245.

[29] Johnson W.L., Cheng Y.T., van Rossum M. et Nicolet M.-A., Nucl. Instr. Meth. B 7/8 (1985) 657.

[30] Workman T.W., Cheng Y.T., Johnson W.L. et Nicolet M.-T., Appl. Phys. Lett. 50 (1987) 1485.

[31] Cheng Y.-T., Dow A.A. et Clemens B.M., Appl. Phys. Lett. 53 (1988) 1346.

[32] Niessen A.K., de Boer F.R., Boom R., de Châtel P.F., Mattens W.C.M. et Miedema A.R., CALPHAD 7 (1983) 51.

[33] Chen Q., Onellion M. et Wall A., Thin Solid Films 196 (1991) 103.

[34] Kief M.T. et Egelhoff Jr W.F., Phys. Rev. B 47 (1993) 10785.

[35] Bauer E., Appl. Surf. Sci. 11/12 (1982) 479.

[36] Miedema A.R., Z. Metallkde. 69 (1978) 455.

[37] de la Figuera J.J., Prieto J.E., Ocal C. et Miranda R., Surf. Sci. 307/309 (1994) 538.

[38] Hofmann S., Surf. Interface Anal. 2 (1980) 148. 